Zeitschrift für Kristallographie, Bd. 136, S. 273-281 (1972)

\title{
Refinement of the structure of anatase at several temperatures*
}

\author{
By M. HorN ** and C. F. SCHWERDTFEGER \\ Department of Physics \\ and E. P. MEAgher \\ Department of Geology \\ University of British Columbia, Vancouver, Canada
}

(Received 5 May 1972)

\begin{abstract}
Auszug
Die Kristallstruktur von Anatas wurde aus Interferenzdaten, erhalten mittels Weissenberg-Methoden bei verschiedenen Temperaturen zwischen $25^{\circ} \mathrm{C}$ und $800^{\circ} \mathrm{C}$, verfeinert. Der einzige Atomorts-Parameter, $z_{0}$, ändert sich gleichmäßig von $0,2081(2)$ bei $25^{\circ} \mathrm{C}$ bis $0,2076(3)$ bei $800^{\circ} \mathrm{C}$. Diese Änderung ist um eine Größenordnung kleiner als der Wert, den HoRN und ScHWERDTFEGER zur Erklärung der Temperatur-Abhängigkeit der paramagnetischen ElektronenResonanz 1971 vorgeschlagen hatten. Die Wärmeschwingungen der Ti-Ionen sind isotrop, die der O-Ionen stark anisotrop. Die Möglichkeit, daß die thermischen Verschiebungen nennenswerte Korrekturen in den Atomabstands-Berechnungen bedingen, wird diskutiert.
\end{abstract}

\begin{abstract}
The crystal structure of anatase was refined at several temperatures between $25^{\circ} \mathrm{C}$ and $800^{\circ} \mathrm{C}$ using single-crystal $\mathrm{x}$-ray diffraction data. The only structural parameter, the $z$ coordinate of oxygen, changes regularly from $0.2081(2)$ at $25^{\circ} \mathrm{C}$ to $0.2076(3)$ at $800^{\circ} \mathrm{C}$. This change is about an order of magnitude smaller than that proposed by HorN and SCHWERDTFEger (1971) to explain the temperature dependence of electron paramagnetic resonance spectra of anatase. The thermal vibrations of the titanium ions are isotropic, those of the oxygen ions highly anisotropic. That these thermal displacements may produce appreciable corrections to the bond-length calculations is discussed.
\end{abstract}

* Research supported by the National Research Council of Canada.

** Present address: Dept. of Physics, Univorsidad Nacional de Ingenieria, Lima, Peru.

Z. Kristallogr. Bd. 136, 3/4 


\section{Introduction}

Anatase is one of the two tetragonal polymorphic forms of titanium dioxide, the other being rutile. Upon heating above a threshold temperature it transforms irreversibly to rutile. This threshold temperature, at atmospheric pressure, is usually about $1000^{\circ} \mathrm{C}$, but can vary from $400^{\circ} \mathrm{C}$ to $1200^{\circ} \mathrm{C}$, depending on grain size, atmosphere and nature and amount of impurities (Shannon and PASK, 1965).

The structure of anatase was originally determined by VEGARD (1916). Recently, a least-squares refinement of the structure, utilizing powder data, was carried out by Cromer and Herrington (1955). Their results are included in Table 2. RAo et al. (1970) determined the thermal expansion of anatase between $28^{\circ} \mathrm{C}$ and $712^{\circ} \mathrm{C}$; however, no structure investigations of anatase have been undertaken at elevated temperatures.

Electron paramagnetic resonances of regular substitutional $\mathrm{Fe}^{3+}$, and of $\mathrm{Fe}^{3+}$ combined with an oxygen vacancy at a nearest-neighbour site, in anatase show an unusually strong temperature dependence (HoRx and SchWERDTFEGER, 1971). The different aspects of this temperature dependence had been explained consistently by assuming that the oxygen ions shift about $0.04 \AA$ along the $c$ axis with a temperature increase from room temperature to $600^{\circ} \mathrm{C}$. In an effort to confirm this experimentally, high-temperature crystal-structure refinements of anatase were carried out at temperatures of $25,300,600$, $800^{\circ} \mathrm{C}$, using single-crystal $\mathrm{x}$-ray techniques.

\section{Experimental}

The specimens used in this study were transparent yellow crystals from Binntal, Wallis, Switzerland. An electron microprobe analysis showed the sample to be nearly pure $\mathrm{TiO}_{2}$ with a maximum of approximately 0.2 weight percent Fe.

The systematic absences of reflections in Weissenberg photographs confirmed the space group assignment $I 4_{1} /$ amd. The unit-cell parameters at room temperature, $a=3.7842(13) \AA$ and $c=9.5146(15) \AA$, were obtained by a least-squares refinement of back-reflection Weissenberg data. These parameters compare favourably with those determined at room temperature by RAO et al. (1970): $a=3.7845(1) \AA$ and $c=9.5143(4) \AA$. The cell parameters determined by RAo $e t$ al. (1970), interpolated and extrapolated for the temperatures used in this investigation, were therefore used and are given $\mathbf{n}$ Table 1. $\mathrm{RAO}_{\mathrm{AO}}$ et al. indicated the errors of the cell parameters only at room 
Table 1. Lattice parameters of anatase

\begin{tabular}{|c|c|c|}
\hline$T$ & $a$ & $c$ \\
\hline $25^{\circ} \mathrm{C}$ & $3.7842(13) \AA$ & $9.5146(15) \AA$ present work \\
\hline 25 & $3.7845(4)$ & $9.514(1)$ \\
\hline 300 & $3.7892(4)$ & $9.537(1)$ \\
\hline 600 & $3.7971(4)$ & NAO el Cal. (19RO) \\
\hline 800 & $3.8040(8)$ & $9.614(2)$ \\
\hline
\end{tabular}

temperature. The errors of the cell parameters, as stated in Table 1 and used in the calculation of the errors of the bond lengths, are estimated from the errors at room temperature and the scattering of the experimental points on a plot of the cell parameters versus temperature.

For the final intensity measurements a small, wedge-shaped cleavage fragment was mounted in a tapered silica capillary for rotation about the $a$ axis. The largest diameter of the crystal was ca. $0.09 \mathrm{~mm}$, the smallest ca. $0.03 \mathrm{~mm}$ and its volume $1.0 \times 10^{-4} \mathrm{~mm}^{3}$.

Three-dimensional intensity data were collected utilizing flat-cone geometry on a manual Weissenberg scintillation-counter diffractometer. Zirconium-filtered Mo radiation was used in conjunction with a pulse-height discriminator. Only those reflections having $(\sin \theta) / \lambda$ $\leq 0.55$ were recorded on a strip-chart recorder and their intensities measured with a planimeter. The flat-cone geometry was required by the furnace which is a modified version of a design by Fort and Peacor (1967).

Fifty six nonequivalent reflections were recorded at temperatures of $25,300,600$ and $800^{\circ} \mathrm{C}$, the $25^{\circ} \mathrm{C}$ measurements being repeated after the heating cycle. These temperatures, measured with a thermocouple in place of the sample, are estimated to be accurate to $\pm 5^{\circ} \mathrm{C}$.

In order to estimate the absorption in the crystal, all 210 reflections, including symmetry-equivalent ones, were measured at room temperature. The intensities of equivalent reflections differed as much as $20 \%$, indicating appreciable $x$-ray absorption in the sample. The measured intensities were corrected for absorption using a linearabsorption coefficient of $\mu=57.5 \mathrm{~cm}^{-1}$. The calculated transmission factors for all reflections varied between 0.76 and 0.92 ; for the set of nonequivalent reflections used in the refinement they varied between 0.82 and 0.92. Differences of the absorption-corrected intensities of equivalent reflections were smaller than $5 \%$, comparable with the experimental error of the overall system. 


\section{Structural refinements}

Since the Ti ions in anatase occupy special position $4 a$, with point symmetry $\overline{4} 2 m$, and the oxygen ions occupy special position $8 e$, with point symmetry $m m$, only one structural parameter, the $z$ coordinate of oxygen, need be determined.

The intensities, corrected for absorption and the Lorentz and polarization factor, were submitted to a full-matrix least-squares refinement, using a modified version of the computer program ORFLS (Busnng et al., 1962). Since the bonds in rutile are highly ionic (LADD, 1969), and since anatase is quite similar to rutile (the structural differences are only in the second-nearest neighbour coordination), form factors for $\mathrm{Ti}^{4+}$ (International tables, 1962) and $\mathrm{O}^{2-}$ (SUZUKI, 1960) were used.

Starting parameters for the refinements were those given by Cromer and Herrington (1955) as included in Table 2. In the initial refinements a comparison of measured and calculated structure factors showed that, for all large structure factors, $F_{\mathrm{o}}<F_{\mathrm{c}}$. An extinction correction was then applied to the data in the least-squares refinement by minimizing the function $\underset{i}{\sum_{i}}\left[F_{\mathrm{o}, i}-S \cdot F_{\mathrm{c}, i} /\left(1+\alpha \cdot I_{i}\right)\right]^{2}$, where $I_{i}$ are the uncorrected intensities, $\alpha$ the extinction coefficient, adjusted by the least-squares calculation, $S$ the scale factor and $w_{i}$ the weights. The weighting scheme of CRUICKSHANK (1965) was applied.

In the final refinements anisotropic temperature factors were used. The special positions of the titanium and oxygen ions imply that the principal axes of the thermal ellipsoids coincide with the crystallographic axes for the two ions; therefore all $B_{i j}=0$ for $i \neq j$, and in addition for titanium, $B_{11}=B_{22}$. The parameters to be adjusted by the least-squares calculation were therefore: the oxygen parame-

Table 2. Oxygen parameter $z$ and isotropic equivalents of anisotropic temperature factors of anatase

\begin{tabular}{c|c|c|c}
\hline$T$ & $z$ & $B_{\mathrm{o}}$ & $B_{\mathrm{T} 1}$ \\
\hline$*$ & $0.2064(9)$ & $0.664(158)$ & $0.510(96)$ \\
$25^{\circ} \mathrm{C}$ & $0.2081(2)$ & $0.613(90)$ & $0.390(63)$ \\
300 & $0.2079(2)$ & $0.951(90)$ & $0.636(58)$ \\
600 & $0.2078(2)$ & $1.483(101)$ & $1.030(60)$ \\
800 & $0.2076(3)$ & $1.870(137)$ & $1.323(78)$
\end{tabular}

* Cromer and Herrington (1955), room temperature. 
Table 3. Root-mean-squares of thermal displacements along the principal axes in anatase

The $x$ axis of the thermal vibrations ellipsoid of the oxygen ion is perpendicular to the (100) plane formed by the three nearest $\mathrm{Ti}$ neighbours, and all principal axes coincide with the crystallographic axes

\begin{tabular}{c|c|c|c|l|l}
\hline \multirow{2}{*}{$T$} & \multicolumn{3}{|c|}{ Oxygen } & \multicolumn{2}{c}{ Titanium } \\
\cline { 2 - 6 } & $x$ & \multicolumn{1}{|c|}{$y$} & $z^{*}$ & \multicolumn{1}{c}{$x=y$} & \multicolumn{1}{c}{$z$} \\
\hline $25^{\circ} \mathrm{C}$ & $.0 .096(6) \AA$ & $0.080(8) \AA$ & $0.088(6) \AA$ & $0.070(5) \AA$ & $0.070(3) \AA$ \\
300 & $0.124(5)$ & $0.096(6)$ & $0.107(5)$ & $0.090(5)$ & $0.090(3)$ \\
600 & $0.165(5)$ & $0.115(6)$ & $0.126(4)$ & $0.116(4)$ & $0.112(2)$ \\
800 & $0.185(6)$ & $0.112(7)$ & $0.156(4)$ & $0.129(5)$ & $0.131(2)$
\end{tabular}

* $z$ is the fractional coordinate corresponding to Niggir's $p$.

ter $z^{*}$, three anisotropic temperature factors $B_{i j}$ for oxygen, two for titanium, the scale factor $S$, and the extinction coefficient $\alpha$.

The results are summarized in Tables 2 and 3 , including the standard deviations. Table 2 gives the oxygen parameter $z$, assuming the coordinate origin at $\overline{4} \mathbf{2}$, and isotropic equivalents of the anisotropic temperature factors for $\mathrm{Ti}$ and $\mathrm{O}$. Table 3 gives the anisotropic thermal parameters in the form of root-mean-squares of thermal displacements along the principal axes. The $x$ axis of the thermalvibration ellipsoid of the oxygen ion is perpendicular to the (100) plane formed by the three nearest $\mathrm{Ti}$ neighbours. The weighted $R$ values in these refinements are between 0.022 and 0.024 for all four temperatures. The results of the structure refinements at room temperature before and after heating are the same within one standard deviation.

In addition, least-squares refinements were carried out under different conditions such as: different starting parameters, no absorption correction, no extinction correction, different weighting schemes, form factors of neutral oxygen and titanium, and isotropic temperature factors. In most of these calculations the standard error of the oxygen parameter, as well as the $R$ value, were larger, but the final results, as given in Table 2, were always included within one standard deviation of the results obtained with these modified least-squares calculations.

\section{Discussion of the structure}

The general features of the anatase structure have been established by previous workers (see Bragg and ClaringBull, 1966). They are in agreement with the results of the present work. Basically, the 
Table 4. Interionic distances and angles in anatase

Values are calculated from the mean positions of the ions

\begin{tabular}{c|l|l|l|l|l|l}
\hline$T$ & $\mathrm{Ti}-\mathrm{O}$ & $\begin{array}{c}\mathrm{Ti}-\mathrm{O} \\
\|[001]\end{array}$ & $\begin{array}{c}\mathrm{O}-\mathrm{O} \\
\text { shared } \\
\text { edge }\end{array}$ & $\mathrm{O}-\mathrm{O}$ & $\begin{array}{c}\mathrm{O}-\mathrm{O} \\
\text { and nearest } \\
\mathrm{Ti}-\mathrm{Ti}\end{array}$ & $\begin{array}{c}\text { angle } \\
(\mathrm{O}-\mathrm{Ti}-0)\end{array}$ \\
\hline $25^{\circ} \mathrm{C}$ & $1.9338(5) \AA$ & $1.9797(23) \AA$ & $2.4658(29) \AA$ & $2.7924(13) \AA$ & $3.0394(2) \AA$ & $101.90(7)^{\circ}$ \\
300 & $1.9365(5)$ & $1.9831(22)$ & $2.4681(29)$ & $2.7968(13)$ & $3.0453(2)$ & $101.96(7)$ \\
600 & $1.9410(5)$ & $1.9909(23)$ & $2.4745(30)$ & $2.8037(14)$ & $3.0560(2)$ & $102.01(7)$ \\
800 & $1.9452(7)$ & $1.9957(31)$ & $2.4777(39)$ & $2.8108(18)$ & $3.0650(5)$ & $102.10(9)$
\end{tabular}

structure of anatase can be seen as a framework of distorted $\mathrm{TiO}_{6}$ octahedra sharing four edges. There are two different nearest-neighbour $\mathrm{Ti}-\mathrm{O}$ distances; the two $\mathrm{Ti}-\mathrm{O}$ bonds along the $c$ axis are longer than the four other $\mathrm{Ti}-\mathrm{O}$ bonds. Each oxygen ion is coordinated to three titanium ions, lying together with the oxygen ion in a (100) plane.

The oxygen parameter $z$, as dete mined by us at room temperature, differs by two standard deviations from the value stated by CROMER and Herrington. This difference may have arisen through the use of powder data by the latter investigators.

'Table 2 shows that the oxygen coordinate $z$ changes regularly as a function of temperature. The change is, however, quite small: over the whole analyzed temperature range from $25^{\circ} \mathrm{C}$ to $800^{\circ} \mathrm{C}, z$ decreases 0.0005 , which corresponds to two standard errors of $z$. This change of $z$, equivalent to a shift of $0.005 \AA$ of the oxygen ions along the $c$ axis, is in the direction of increasing distortion of the $\mathrm{TiO}_{6}$ octahedra with increasing temperature.

With the computer program ORFFE (Busing et al., 1964) the distances and angles between the mean positions of the ions were calculated, including their standard errors. The results are given in Table 4, which shows all nearest and second-nearest neighbour distances. Because the temperature dependence of the oxygen parameter $z$ is small, the temperature-induced changes of the interionic distances and angles (not corrected for thermal vibrations) are mainly governed by the lattice expansion. However, two interesting features should be noted: first, the shared octahedral edges increase with temperature less than all the other distances and their increase is less than the relative thermal expansion of the lattice parameters; second, none of the distances increases with temperature as much as the cell edge $c$. 
These observations, however, do not necessarily follow if one considers mean interionic distances, averaged over the thermal displacements, rather than the distances between the mean positions of the ions. To calculate these mean distances one has to know the correlations between the thermal displacements of the ions. If these correlations are unknown, only lower and upper bounds of the thermally corrected bond lengths can be calculated (BUSING and LEVY, 1964). Two particular forms of correlation between the thermal displacements of two atoms are sometimes used to estimate the thermal correction of the interatomic distance: independent thermal displacements of the atoms, which results in a bond length just halfway between the upper and the lower bound, or "riding" motion of one atom on the other, which results in a bond length nearer to the lower bound.

For anatase no data are available to calculate, or even estimate, the predominant vibrational modes. Therefore only the lower and upper bounds of the thermally corrected bond lengths can be calculated. Figure 1 shows these bounds for the nearest-neighbour Ti-O

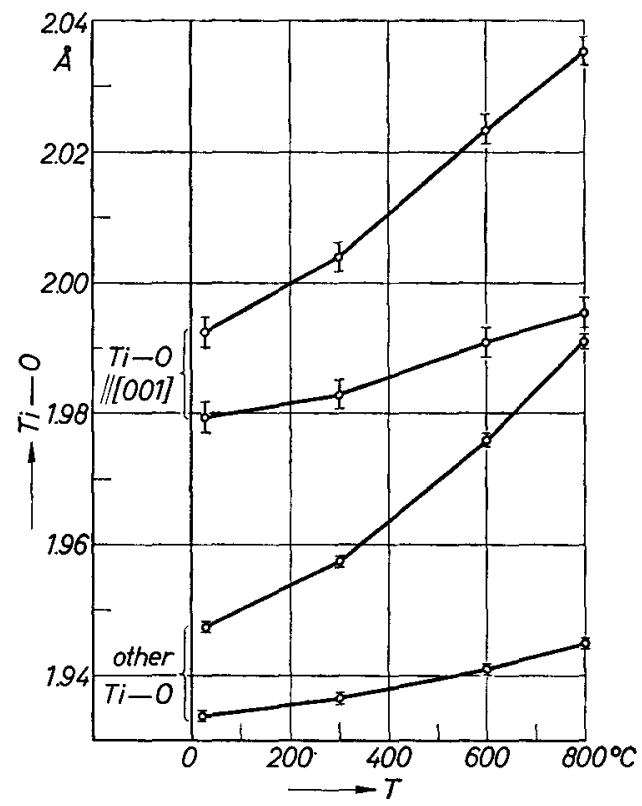

Fig. 1. Upper and lower bounds of the nearest neighbour $\mathbf{T i}-\mathrm{O}$ distances in anatase, corrected for thermal displacements. The lower bounds are equal to the uncorrected bond lengths within the experimental error 
distances. The lower bounds are equal to the uncorrected bond lengths within the experimental error. As can be seen, the corrections may be appreciable. In addition, correlations between the thermal vibrations are imaginable that would bring one of the two bond lengths near to the lower bound, the other to the upper. It is therefore possible that the difference between the two Ti-O distances, corrected for thermal motion, could increase or decrease with temperature. The dynamical state of the crystal may also change with temperature; the correction for a particular bond length would then be a function of temperature.

\section{Conclusions}

The only structural parameter of anatase, the $z$ coordinate of oxygen, changes with temperature regularly but slowly. This same behaviour is therefore reflected in its different interionic distances and angles. Quantitative statements on the temperature-induced changes of the interionic distances can be made, however, only for the distances between the mean positions of the ions. In order to calculate the true mean distances, one requires additional information on the lattice dynamics of anatase. As this information is not available, only lower and upper bounds for the bond lengths can be given.

Since the anatase-rutile transformation is obtained through a reconstructive first-order transition, no feature of the structural changes of anatase with increasing temperature can indicate the approaching transformation to rutile. However, the large thermal vibrations of the oxygen ions out of their bond planes may induce this transformation.

The present crystal-structure study of anatase was undertaken to correlate its results with previous electron paramagnetic resonance investigations of the same mineral. The main result in this connection is the fact that the temperature dependence of the electron paramagnetic resonance spectra of $\mathrm{Fe}$ impurities in anatase cannot be understood from a structural point of view, at least inasmuch as the structure is defined by the mean positions of the ions, determined by $x$-ray diffraction. The change of the oxygen parameter with increasing temperature is in the same direction as deduced from electron paramagnetic resonance data; its magnitude is, however, about an order of magnitude too small to be in agreement with the structural model proposed to explain the electron paramagnetic resonance data (HORN and SCHWERDTFEGER, 1971). 


\section{Bibliography}

L. Bragg and G. Claringbull (1966), The crystalline state. Vol. IV, p. 107, Cornell University Press, New York.

W. R. Busing and H. A. LeVy (1964), The effect of thermal motion on the estimation of bond lengths from diffraction measurements. Acta Crystallogr. $17,142-146$.

W. R. Busing, K. O. Martin and H. A. Levy (1962), ORFLS, a FORTRAN crystallographic least squares program. Oak Ridge National Laboratory, No. ORNL-TM-305.

W. R. Busing, K. O. Martin and H. A. Levy (1964), ORFFE, a FORTRAN crystallographic function and error program. Oak Ridge National Laboratory, No. ORNL-TM-306.

D. T. Cromer and K. Herrington (1955), The structures of anatase and rutile. J. Amer. Chem. Soc. 77, 4708-4709.

D. W. J. Cruickshank (1965), Errors in least squares methods, in computing methods in crystallography, edited by J. S. Rolletr, Pergamon Press.

F. F. Foit and D. R. Peacon (1967), A high temperature furnace for a single crystal x-ray diffractometer. J. Sci. Instrum. 44, 183-185.

M. HorN and C. F. SchwerdTFeger (1971), EPR of substitutional and charge compensated $\mathrm{Fe}^{3+}$ in anatase $\left(\mathrm{TiO}_{2}\right)$. J. Physics Chem. Solids 32, 2529-2538.

International tables for $x$-ray crystallography (1962), Vol. III, p. 204. The form factors of $\mathrm{Ti}-\mathrm{Ti}^{3+}$ quoted there were extrapolated for $\mathrm{Ti}^{4+}$.

M. F. LADD (1969), Bond type in the dioxides of titanium, germanium and tin. Acta Crystallogr. A 25, 486-487.

K. Rao, N. Naidu and L. Iyenga (1970), Thermal expansion of rutile and anatase. J. Amer. Ceram. Soc. 53, 124-126.

R. Shannon and J. PASK (1965), Kinetics of the anatase-rutile transformation. J. Amer. Ceram. Soc. 48, 391-398.

T. Suzuri (1960), Atomic scattering factor for $\mathrm{O}^{2-}$. Acta Crystallogr. 13, 279.

L. VeGard (1916), Results of crystal analysis. Phil. Mag. 32, 505-515. 